\title{
Microfluidic Assays for DNA Manipulation Based on a Block Copolymer Immobilization Strategy
}

\author{
Andreas E. Vasdekis, ${ }^{, \dagger}$ Conlin P. O’Neil, ${ }^{\ddagger}$ Jeffrey A. Hubbell, ${ }^{\ddagger}$ and Demetri Psaltis ${ }^{\dagger}$ \\ Optics Laboratory, School of Engineering and Laboratory of Regenerative Medicine and Pharmacobiology, \\ Institute of Bioengineering, Ecole Polytechnique Fédérale de Lausanne (EPFL), Switzerland
}

Received December 21, 2009; Revised Manuscript Received January 27, 2010

\begin{abstract}
Methods to manipulate and visualize isolated DNA and oligonucleotide strands are important for investigation of their biophysics as well as their interactions with proteins. Herein, we report such a method by combining a block copolymer surface functionalization strategy with microfluidics. The copolymer poly(L-lysine-graftpolyethylene glycol) (PLL- $g$-PEG) coated one surface of the microfluidic channels, rendering it passive to adsorption and thus minimizing any noise arising from nontargeted adsorbed molecules. Single $\lambda$-phage DNA molecules were immobilized and were extended by molecular combing. Their extension did not exceed their contour length, which we attribute to the low surface tension of the coated surface. To demonstrate further the applicability of our method, the anchored DNA was extended by hydrodynamic flow. We propose this method for exploring DNA-protein interactions due to the copolymer's enhanced capacity for single-molecule detection, stability under wet or dry conditions, hydrophilicity, full compatibility with microfluidics and simplicity being a one-step process.
\end{abstract}

\section{Introduction}

Manipulating individual DNA molecules has attracted significant attention the past few years because it enables direct interrogation of single macromolecules and events occurring on their backbone. Typical problems examined by such platforms involve the elastic properties of isolated nucleic acids, ${ }^{1,2}$ sequencing, ${ }^{3,4}$ viral ejection, ${ }^{5,6}$ physical mapping, ${ }^{7}$ and proteinDNA interactions for transcription, replication, and repair. ${ }^{8-11}$ Especially in the cases of mapping and enzymatic activity, resolution can be greatly enhanced by stretching the DNA, usually by anchoring one end and applying an extension force. To this end, a plethora of technologies have been developed, such as the atomic force microscopy, ${ }^{12}$ optical/magnetic tweezers, ${ }^{13,14}$ electrostretching, ${ }^{15}$ and fluidic systems that exert hydrodynamic and capillary forces or impose entropic confinement. ${ }^{16-18}$ Particularly noteworthy are microfluidic approaches, where the possibilities of reconfigurable physiological conditions, the reduced reagent consumption, and potential for multiplexed measurements have sparked renewed interest. ${ }^{19-21}$

A common need to most aforementioned methodologies is the ability to detect single molecules. This ultimate sensitivity level is enabled by high-performance cameras and optics. ${ }^{22} \mathrm{~A}$ more recent approach to enhance the signal-to-noise ratio (SNR) of single molecule techniques is to render the surfaces of interest inert, while maintaining the high affinity and integrity of the target. ${ }^{17}$ In this way, nonspecific interactions are suppressed, and the majority of the signal stems from the targeted bioentity. Characteristic methods to this end involve coatings based on BSA (bovine serum albumin), polyelectrolyte multilayer films, linear and star polyethylene-based polymers (PEGs, PEOs, etc.), and lipid bilayers and vesicles. ${ }^{17,23-25}$ PEG, either by itself $^{26,27}$ or as a block copolymer, ${ }^{28,29}$ has been widely employed as surface coatings largely because of the ease of

\footnotetext{
* Corresponding author. Tel: +41 2169 33742. Fax: +41 216936930. E-mail: andreas.vasdekis@epfl.ch.

Optics Laboratory, School of Engineering.

Laboratory of Regenerative Medicine and Pharmacobiology, Institute of Bioengineering.
}

chemical modification, low protein adsorption properties, low cost, and commercial availability. PLL- $g$-PEG is one such copolymer. It comprises the water-soluble polymer poly(ethylglycol) (PEG), grafted onto the polycationic poly(L-lysine) (PLL) ${ }^{28}$ The PLL is positively charged and hence adsorbs the copolymer on negatively charged surfaces. When the coated surface is hydrated, the PEG forms a dense brush and acts as the blocking agent, preventing bioentities from adsorbing nonspecifically to the surface because of steric stabilization. For its superior protection from nonspecific adsorption and capacity to form large-area uniform coatings, PLL- $g$-PEG has found several successful applications in molecular sensing and in cell cultures. $^{30-32}$

In this study, we report the manipulation and visualization of single DNA molecules by combining microfluidics with the block copolymer PLL-g-PEG. Bacteriophage $\lambda$-DNA was stretched by both molecular combing and hydrodynamic flow in the same microfluidic chip. Apart from its enhanced capacity for single molecule detection, PLL- $g$-PEG was chosen for its stability under ambient conditions, a significant advantage for molecular combing, where the surfaces are dried and subsequently rehydrated for imaging. A $\mathrm{SiO}_{2}$ coverslip was bonded with a polydimethylsiloxane (PDMS) channel and then functionalized, exhibiting full compatibility between our immobilization method with microfluidics and the associated $\mathrm{O}_{2}$ plasma processing. The hydrophilic nature of the PEG brush reduced the surface tension, thus stretching the DNA only below its contour length during molecular combing. Sequence-dependent immobilization was achieved by hybridizing the $\lambda$-DNA with a biotinylated oligonucleotide that interacted with the surface via an avidin-biotin bond.

\section{Experimental Section}

Microfluidic Chips. The microfluidic chip was realized in PDMS on a $\mathrm{SiO}_{2}$ coverslip (Figure 1b). The PDMS layer was prepared by replica molding. The master mold was defined using standard UV lithography on a $15 \mu \mathrm{m}$ thick (unless stated otherwise) SU-8 film (GM1070, spin speed of $4800 \mathrm{rpm}$, Gersteltec, Switzerland) on a silicon 
(a)

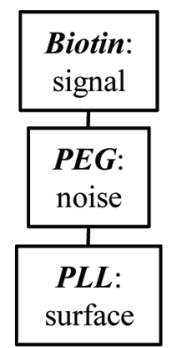

(b)

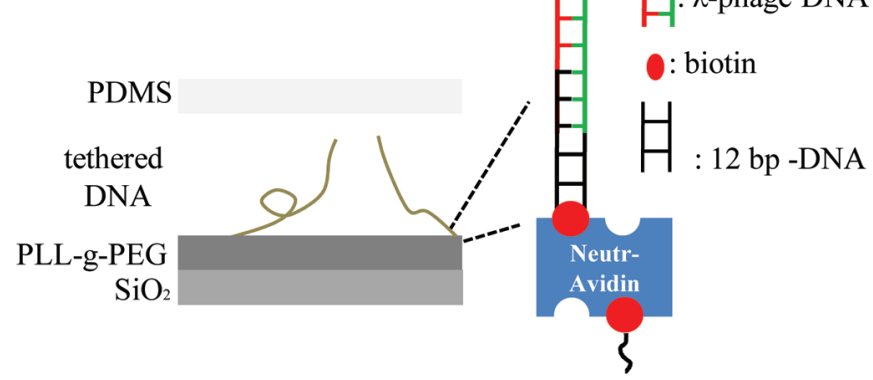

Figure 1. (a) Block diagram illustrating the three functionalities of the PLL-g-PEG, namely surface: attraction, noise repulsion, and signal reception. (b) Schematic of the surface architecture.

wafer. The structured SU-8 was treated with trimethylchlorosilane vapor (TMS, Sigma Aldrich, Switzerland), and the pattern was transferred into the PDMS (Dow Corning Sylgard 184, Omya AG, Switzerland). The coverslips (Menzel Gläser, Germany) and PDMS fluidic channels were treated with $\mathrm{O}_{2}$ plasma $(12 \mathrm{~W}, 20 \mathrm{~s})$ and brought in contact to bond permanently. The fluidic interconnect was realized through Tygon tubing (Cole Palmer via Fisher Scientific AG, Switzerland), and the flow rate was controlled either manually or via a syringe pump (New Era Pump Systems, Wantagh, NY).

Surface Preparation. The $\mathrm{SiO}_{2}$ coverslips were thoroughly cleaned by sonicating in methanol and acetone baths in concentrated nitric acid (65\%) and $6 \mathrm{M} \mathrm{KOH} \mathrm{(Sigma} \mathrm{Aldrich,} \mathrm{Switzerland)} \mathrm{and} \mathrm{were} \mathrm{stored}$ until use in Milli-Q water. All steps lasted 35 min and were separated by multiple Milli-Q water rinses. Prior to bonding, the substrates were further cleaned in $\mathrm{O}_{2}$ plasma $(12 \mathrm{~W})$ for $20 \mathrm{~min}$. The employed copolymer was the PLL(20)-g[3.6]-PEG(2) (Susos AG, Switzerland), a PLL-g-PEG based on a $20 \mathrm{kDa}$ PLL with an average of 1 out of 3.6 lysine side chains grafted with a $2 \mathrm{kDa}$ PEG chain. $17 \%$ of the PEG(2) chains had been substituted by a biotinylated PEG(3.4) chain. ${ }^{30}$ The PLL-g-PEG was dissolved at a concentration of $1 \mathrm{mg} / \mathrm{mL}$ in a $10 \mathrm{mM}$ HEPES solution (Sigma Aldrich, Switzerland) adjusted to $\mathrm{pH}$ 7.4. The solution was filtered through a $0.2 \mu \mathrm{m}$ Durapore membrane (Sigma Aldrich, Switzerland). When the microfluidic chip was assembled, the copolymer solution was introduced into the chip and allowed to adsorb on the surface for $45 \mathrm{~min}$. During this step, we expect most of the copolymer to be adsorbed on the glass surface. ${ }^{33}$ Subsequently, the microfluidic channels were rinsed with $10 \mu \mathrm{L}$ of Milli Q water and were incubated with NeutrAvidin (30 nM, Pierce Biotechnologies, Switzerland) for 30 min. ${ }^{17}$ The excess protein was washed away with $10 \mu \mathrm{L}$ of Milli Q water, and the microfluidic channel was incubated with the biotinylated DNA solution for $\sim 1 \mathrm{~h}$ under no external pressure.

DNA Preparation. Biotinylated oligonucleotides were ligated at the cohesive $3^{\prime}$ end of double-stranded bacteriophage $\lambda$-DNA ( $48.5 \mathrm{~kb}$, New England Biolabs, Ipswich, MA). The oligonucleotide sequence was $5^{\prime}$-p ggg cgg cga cet-biotin (Microsynth AG, Switzerland). First, the $\lambda$-DNA and the oligonucleotides were mixed at a molar ratio of $1: 14$, heated at $80{ }^{\circ} \mathrm{C}$ for $10 \mathrm{~min}$, and cooled at room temperature for over $1 \mathrm{~h}$. Ligation took place by the addition of the DNA ligase, cooling of the mixture to $16^{\circ} \mathrm{C}$ for $2 \mathrm{~h}$, and then heating the mixture to $65{ }^{\circ} \mathrm{C}$ to inactivate the ligase. The biotinylated product was stored in $1 \times \mathrm{TE}$ buffer (10 mM Tris pH 8, 1 mM EDTA, Ambion, Applied Biosystems, Switzerland) at $-20{ }^{\circ} \mathrm{C}$ until used. The ligation product was not columnpurified. For direct imaging, the DNA was stained with the intercalating dye Yoyo-1 iodine (Invitrogen, Switzerland) at a bp/dye ratio of 5:1 by incubation at $4{ }^{\circ} \mathrm{C}$ for $1 \mathrm{~h}$. The biotinylation was confirmed by mixing the ligation product with SuperAvidin-coated microspheres (diameter $9.95 \mu \mathrm{m}$, Bangs Laboratories, Fishers, IN) at an approximate ratio of $10^{8}$ spheres for $6 \mathrm{ng}$ of DNA and measuring the diffusion of the stained DNA in the proximity of the spheres (Supporting Information, Figure S1). The stained biotinylated DNA solution used in the force spectroscopy and molecular combing experiments had a concentrations of 0.262 and $0.131 \mu \mathrm{g} / \mu \mathrm{L}$, respectively.

Imaging and Image Analysis. The experiments were performed on an inverted frame microscope (Olympus IX71, Japan), equipped with a high-resolution stage (MS-2000, Applied Scientific Instrumentation, Eugene, OR) and a $100 \times, \mathrm{NA}=1.45$ objective (PLAPO100XO/ TRIFM-SP, Olympus, Japan). Images and videos were captured with an EMCCD camera (iXon DV885 VP, Andor Technology, Ireland) cooled to $-80^{\circ} \mathrm{C}$. The chromophore Yoyo- 1 was excited with the 488 $\mathrm{nm}$ line of an argon ion laser (Innova 300, Coherent, Santa Clara CA), and the fluorescence was collected through the dichroic and short-pass filters (Z488 RDC and ET500 LP, Chroma Technology, Rockingham, VT). The image pixel size was calibrated by imaging transparent squares of different sizes, which were defined by electron-beam lithography (EBPG 5000, Vistec) on a $200 \mathrm{~nm}$ thick aluminum film on a coverslip. Linear fitting yielded a ratio of actual length over image pixel size equal to $0.0798 \pm 10^{-4} \mu \mathrm{m}$. The images and videos were analyzed through a program written in MATLAB (Mathworks, Switzerland). The data analysis for the DNA length measurement involved the removal of contiguous pixels with intensity substantially higher than the background. ${ }^{34}$ Spatial noise was further removed by convolving each image with a Gaussian of width $0.2 \mu \mathrm{m}^{2}$. This value was determined by imaging through the same optical system single Rhodamine $6 \mathrm{G}$ molecules ( $10^{-11} \mathrm{M}$, Sigma Aldrich), covered by a thin polymethylmethacrylate (PMMA, Sigma Aldrich, Switzerland) film. ${ }^{35}$

\section{Results and Discussion}

Surface Immobilization Strategy. Our objective was to stretch bacteriophage $\lambda$-DNA, anchored on the PLL- $g$-PEG functionalized surface. This method was adopted to minimize the biological recognition of the surface and hence the contribution of the background to the signal. In Figure 1a, a block diagram illustrates the three-fold functionality of the PLL-gPEG. When hydrated, the dense PEG brushes block the nonspecific interactions with the surface via steric stabilization, whereas the PLL acts as the anchor to the surface. ${ }^{28}$ The marked advantages of our method are uniform large-area and uncharged coatings exhibiting enhanced resistance to nonspecific recognition, compatibility with microfluidics, stability under ambient conditions, and low surface tension. The latter is of special note with regards to molecular combing, where care must be taken not to overstretch the molecules. To our knowledge, this is the first report on DNA combing on surfaces that are both hydrophilic and marked with a blocking agent.

In our experiments, the microfluidic chip was assembled and subsequently its bottom surface was coated with a PLL- $g$-PEG monolayer via aqueous chemistry (Figure 1b). In the copolymer solution, a small fraction (17\%) of biotinylated PEGs was 
introduced to immobilize the DNA via a biotin-avidin bond. ${ }^{36}$ NeutrAvidin was then incubated to adsorb on the surface. The adsorbed proteins immobilized the biotinylated $\lambda$-DNA because avidin exhibits four binding sites to the biotin. The immobilization is sequence-specific because the $\lambda$-DNA is hybridized with a biotinylated oligonucleotide. The DNA was extended by both a receding meniscus and hydrodynamic flow. The formation of a DNA-protein complex for DNA extension has been employed in the past; however these reports employed either bilipid surface monolayers, which are not suitable for molecular combing, ${ }^{17}$ or hydrophobic surfaces that nonspecifically attract proteins. ${ }^{37}$

Molecular Combing. In molecular combing, single DNA molecules first bind to the surfaces and subsequently are stretched by a receding meniscus. The molecules are attached on the surface and remain immobile even after rehydration. This is one of the simplest methods for DNA manipulation and was pioneered by Bensimon et al. using silanized surfaces to bind nonspecifically the DNA. ${ }^{38}$ Since then, it has been extensively used in numerous applications, predominately in gene mapping and protein-DNA interactions. Recently, molecular combing has received renewed interest because of its compatibility with microfluidics. ${ }^{21}$ However, the integration of surfaces resistant to nonspecific interactions has received little attention, apart from a recent report employing Coulombic interactions that nevertheless interfere with the extension of the molecule. ${ }^{34,39}$ The major drawback of molecular combing is that the DNA is overstretched into forms longer than its contour length. ${ }^{40}$ The former has been attributed to the high extension force due to the hydrophobic nature of the surface. ${ }^{39}$ To address this, the treatment of the surfaces to lower their surface tension ${ }^{8,39,41}$ and the use of certain protein-DNA complexes ${ }^{37}$ have been proposed.

Our approach to address simultaneously all of these challenges was to coat the bottom surface of the microfluidic chip with PLL-g-PEG. The monolayer remained stable when the surfaces were dried during the combing process and also exhibited a low surface tension due to the hydrophilic nature of the hydrated PEG brushes. ${ }^{42}$ Subsequent to chip realization and functionalization, the DNA solution was introduced and allowed to adsorb. Combing was performed by flowing the remaining solution out of the channel via evaporation. In Figure 2, a typical image of the stretched DNA is illustrated that reached an extension length of $8 \mu \mathrm{m}$. The molecules are aligned parallel to the microfluidic walls (Figure 2, inset), indicating the low curvature of the air-water interface as it passes through the channel. ${ }^{43}$ The extension length distribution of $\lambda$-DNA combed in a microfluidic channel with a cross sectional area of $100 \times 100 \mu \mathrm{m}^{2}$ is shown in Figure 3. The majority of the combed DNA molecules had an extension length between 7 and $11 \mu \mathrm{m}$, whereas the mean extension was $\sim 9 \mu \mathrm{m}$, corresponding to an extension ratio of $\langle x\rangle / L \approx 0.45$, where $L$ is the contour length. The molecules did not stretch further than the contour length, and we attribute this to the block copolymer monolayer that rendered the surface hydrophilic. ${ }^{39,41}$ In addition, random ligation leading to molecules longer than $21 \mu \mathrm{m}$ was $<1 \%$ (Figure 3, inset).

Stretching by Hydrodynamic Flow. A less straightforward method to extend the DNA is to immobilize it on a surface and apply hydrodynamic flow. Under zero flow conditions, the immobilized DNA is kept relatively compact and forms a random coil. By pumping a buffer through the microfluidic channel, a force is applied on the DNA itself, and the macromolecule is extended. Despite the difficulty in determining the flow velocity in the vicinity of the surface ${ }^{40}$ and the

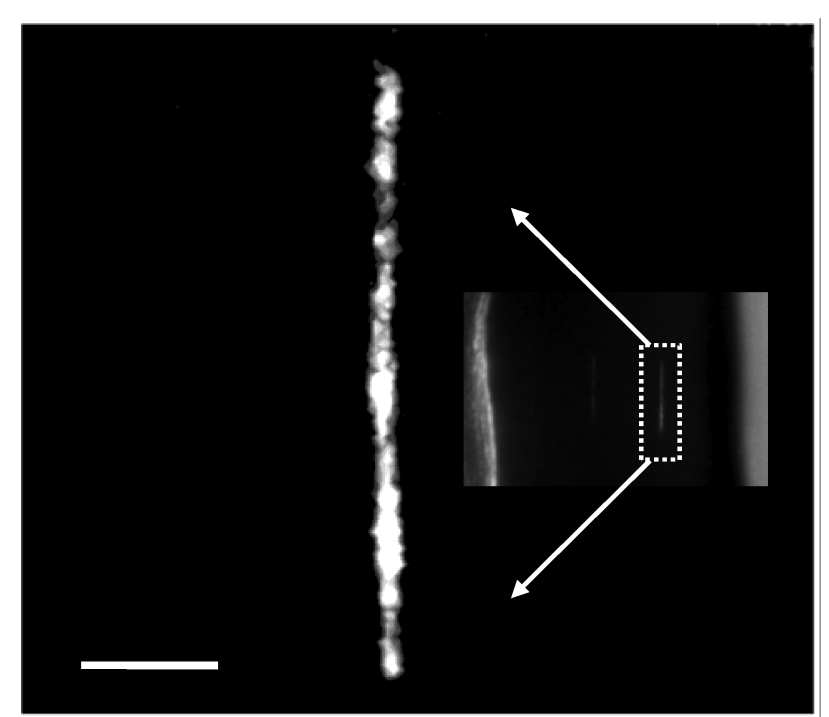

Figure 2. Stretched bacteriophage DNA on a PLL-g-PEG surface by molecular combing. The scale bar is $2 \mu \mathrm{m}$. Inset: the same snapshot at a lower magnification indicating the microfluidic boundaries; the channel cross-sectional dimensions were $40 \times 40 \mu \mathrm{m}^{2}$, and the flow rate was $0.5 \mathrm{~mL} / \mathrm{h}$.

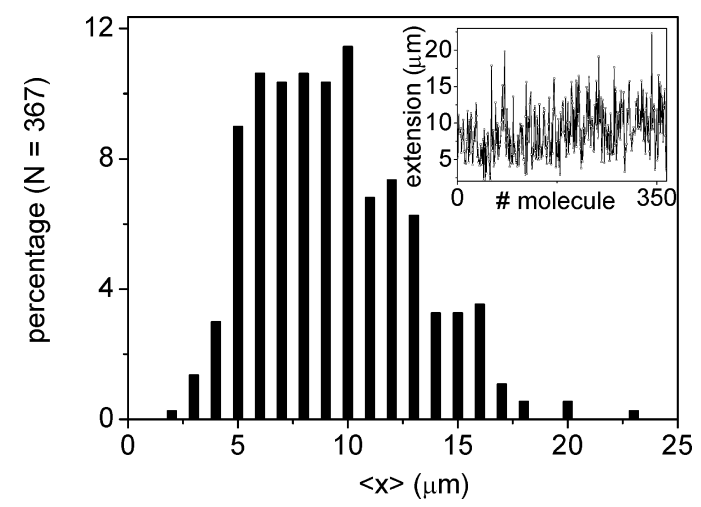

Figure 3. Extension ratio histogram for the DNA stretched by molecular combing on the PLL-g-PEG-coated microfluidic channel. Inset: raw data of 367 molecules.

inhomogeneity of the drag force along the DNA, ${ }^{44}$ multiple assays have been developed using this method, such as the "DNA curtains". 17

To demonstrate further the applicability of our method in performing similar single molecule experiments, we immobilized the biotinylated DNA on the PLL- $g$-PEG coated surface and subsequently applied an extension force via buffer flow. In the absence of flow, the surface produced low signal, comparable to the background intensity, apart from the weak fluorescence of few unstretched tethered molecules (Figure 4a). When buffer flow is applied, the DNA molecules were extended, forming nonordered curtains and allowing their visualization along their contour length (Figure $4 \mathrm{~b}$ ). The repeatability of the method was indicated by the successive buffer flow termination and initiation that resulted in the re-extension of the DNA and by the possibility of extending the DNA in opposite directions by alternating the flow direction (Figure 4c and the Supporting Information). During flow stretching, we did not observe any nonspecific electrostatic interactions between the polycationic PLL and negatively charged DNA. We attribute this to the effective screening of the PLL charge by the $\mathrm{SiO}_{2}$ and to the increased distance of the DNA from the PLL due to the PEG brushes. 

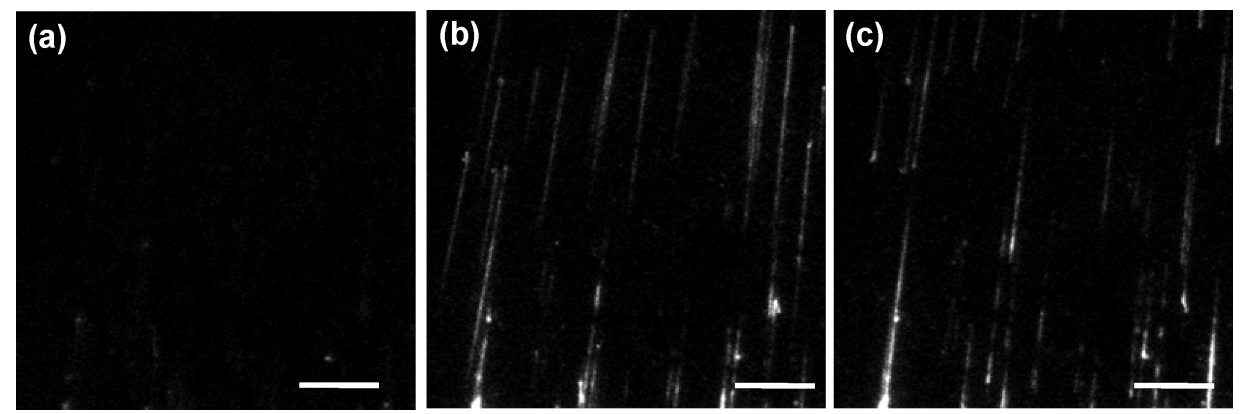

Figure 4. Image of (a) a surface with immobilized DNA under zero flow conditions and (b) the formation of nonordered DNA curtains under maximum extension force pointing in one direction and (c) then in the opposite direction. The scale bar corresponds to $5 \mu \mathrm{m}$.
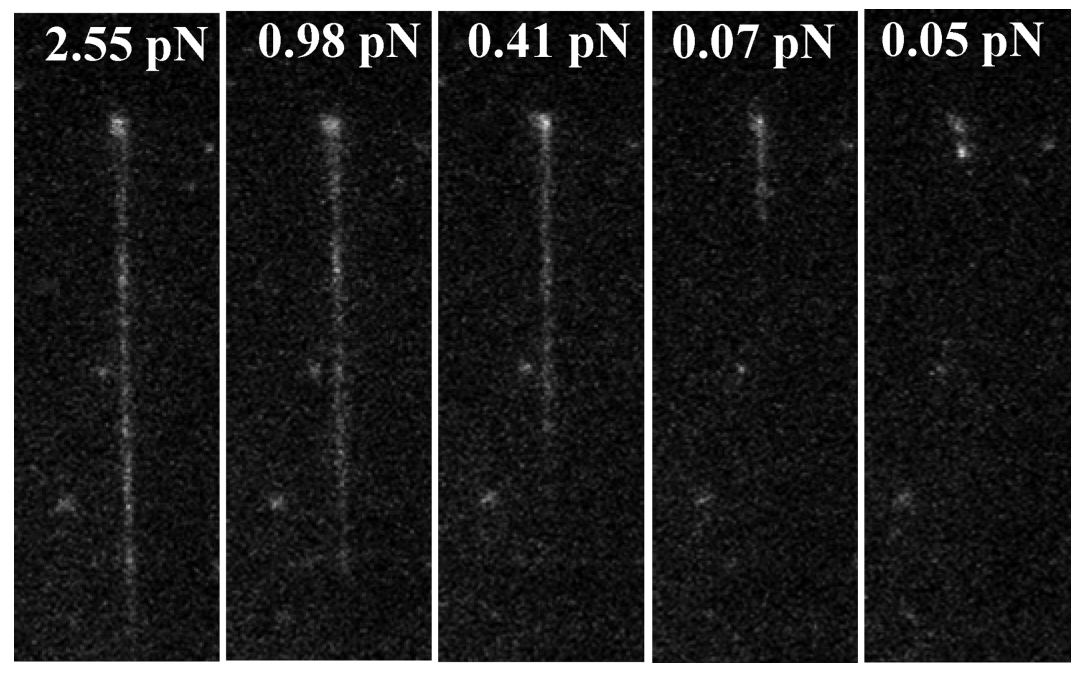

Figure 5. Snapshots of the DNA at different extension lengths experiencing the noted force.

We varied the extension length of the DNA by modifying the extension force through variations of the flow rate inside the microfluidic channel. ${ }^{17}$ The tethered molecules did not lose contact with the surface, even when extension forces up to 6 $\mathrm{pN}$ were applied, proving thus the suitability of our method for manipulating DNA molecules within physiological conditions. In Figure 5, snapshots of DNA molecules experiencing different extension forces are shown. The extension force was determined from the mean extension length, using the polynomial relationship derived from the wormlike chain model (WLC) ${ }^{44}$ For this calculation, the contour and persistence lengths of the stained bacteriophage $\lambda$-DNA were included to be $21 \mu \mathrm{m}$ and $16.8 \mathrm{~nm}$, respectively. ${ }^{45}$

\section{Conclusions}

We have demonstrated the use of the block copolymer PLL$g$-PEG to functionalize one surface of a microfluidic chip and immobilize bacteriophage $\lambda$-DNA at its $3^{\prime}$ end. This functionalization strategy was chosen for its enhanced resistance to protein adsorption, stability under both wet and dry conditions, and simplicity and compatibility with microfluidics. To stretch the DNA, extension forces were applied by both a receding meniscus and hydrodynamic flow. In the case of molecular combing, we found that the majority of the DNA (>99\%) was not overstretched beyond its contour length. This marked advantage makes this immobilization strategy an ideal candidate for exploring DNA-protein interactions. In the case of stretching by hydrodynamic flow, the tethered molecules remained in contact with the surface, even when the molecules were extended to lengths close to their contour length. Currently, we are exploring alternative DNA-protein and protein-copolymer complexes for immobilizing the DNA. ${ }^{37}$ In addition, alternative types of substrates and copolymers are investigated to add more functionalities on the surfaces, such as optical capabilities for interrogating single DNA molecules by optofluidic means. ${ }^{46}$

Acknowledgment. We thank Aleksandra Radenovic and Bart Deplancke for their experimental assistance in DNA hybridization and fruitful discussions.

Supporting Information Available. Immobilized biotinylated DNA molecule on a SuperAvidin coated microsphere at successive frames and flow stretched DNA in two alternating and opposite directions. This material is available free of charge via the Internet at http://pubs.acs.org.

\section{References and Notes}

(1) Bustamante, C.; Marko, J. F.; Siggia, E. D.; Smith, S. Science 1994, $265,1599-1600$.

(2) Quake, S. R.; Babcock, H.; Chu, S. Nature 1997, 388, 151-154.

(3) Braslavsky, I.; Hebert, B.; Kartalov, E.; Quake, S. R. Proc. Natl. Acad. Sci. U.S.A. 2003, 100, 3960-3964.

(4) Tanaka, H.; Kawai, T. Nat. Nanotechnol. 2009, 4, 518-522.

(5) Mangenot, S.; Hochrein, M.; Radler, J.; Letellier, L. Curr. Biol. 2005, $15,430-435$.

(6) Grayson, P.; Han, L.; Winther, T.; Phillips, R. Proc. Natl. Acad. Sci. U.S.A. 2007, 104, 14652-14657.

(7) Michalet, X.; Ekong, R.; Fougerousse, F.; Rousseaux, S.; Schurra, C.; Hornigold, N.; vanSlegtenhorst, M.; Wolfe, J.; Povey, S.; Beckmann, J. S.; Bensimon, A. Science 1997, 277, 1518-1523.

(8) Gueroui, Z.; Place, C.; Freyssingeas, E.; Berge, B. Proc. Natl. Acad. Sci. U.S.A. 2002, 99, 6005-6010.

(9) Lee, J. B.; Hite, R. K.; Hamdan, S. M.; Xie, X. S.; Richardson, C. C.; van Oijen, A. M. Nature 2006, 439, 621-624. 
(10) Graneli, A.; Yeykal, C. C.; Robertson, R. B.; Greene, E. C. Proc Natl. Acad. Sci. U.S.A. 2006, 103, 1221-1226.

(11) Kim, J. H.; Larson, R. G. Nucleic Acids Res. 2007, 35, 3848-3858.

(12) Bustamante, C.; Smith, S. B.; Liphardt, J.; Smith, D. Curr. Opin. Struct. Biol. 2000, 10, 279-285.

(13) Hodges, C.; Bintu, L.; Lubkowska, L.; Kashlev, M.; Bustamante, C. Science 2009, 325, 626-628.

(14) Strick, T. R.; Croquette, V.; Bensimon, D. Nature 2000, 404, 901904.

(15) Bakajin, O. B.; Duke, T. A. J.; Chou, C. F.; Chan, S. S.; Austin, R. H.; Cox, E. C. Phys. Rev. Lett. 1998, 80, 2737-2740.

(16) Tegenfeldt, J. O.; Prinz, C.; Cao, H.; Chou, S.; Reisner, W. W.; Riehn, R.; Wang, Y. M.; Cox, E. C.; Sturm, J. C.; Silberzan, P.; Austin, R. H. Proc. Natl. Acad. Sci. U.S.A. 2004, 101, 10979-10983.

(17) Granéli, A.; Yeykal, C. C.; Prasad, T. K.; Greene, E. C. Langmuir 2006, 22, 292-299.

(18) Persson, F.; Utko, P.; Reisner, W.; Larsen, N. B.; Kristensen, A. Nano Lett. 2009, 9, 1382-1385.

(19) Kim, S. J.; Blainey, P. C.; Schroeder, C. M.; Xie, X. S. Nat. Methods 2007, 4, 397-399.

(20) Brewer, L. R.; Bianco, P. R. Nat. Methods 2008, 5, 517-525.

(21) Sidorova, J. M.; Li, N.; SSchwartz, D. C.; Folch, A.; Monnat, R. J. J. Nat. Protoc. 2009, 4, 849-861.

(22) Lakowicz, J. R. Springer 2006, 797-840.

(23) Koopmans, W. J. A.; Schmidt, T.; van Noort, J. ChemPhysChem 2008, 9, 2002-2009.

(24) Rasnik, I.; McKinney, S. A.; Ha, T. Acc. Chem. Res. 2005, 38, 542 548.

(25) Chan, T. F.; Ha, C.; Phong, A.; Cai, D. M.; Wan, E.; Leung, L.; Kwok, P. Y.; Xiao, M. Nucleic Acids Res. 2006, 34, e113, pages 1-6.

(26) Otsuka, H.; Nagasaki, Y.; Kataoka, K. Curr. Opin. Colloid Interface Sci. 2001, 6, 3-10.

(27) Alcantar, N. A.; Aydil, E. S.; Israelachvili, J. N. J. Biomed. Mater. Res. 2000, 51, 343-351.

(28) Elbert, D. L.; Hubbell, J. A. Chem. Biol. 1998, 5, 177-183.
(29) Kato, K.; Uchida, E.; Kang, E. T.; Uyama, Y.; Ikada, Y. Prog. Polym. Sci. 2003, 28, 209-259.

(30) Kenausis, G. L.; Voros, J.; Elbert, D. L.; Huang, N. P.; Hofer, R.; Ruiz-Taylor, L.; Textor, M.; Hubbell, J. A.; Spencer, N. D. J. Phys. Chem. B 2000, 104, 3298-3309.

(31) Huang, N. P.; Voros, J.; De Paul, S. M.; Textor, M.; Spencer, N. D. Langmuir 2002, 18, 220-230.

(32) Gunnarsson, A.; Jonsson, P.; Marie, R.; Tegenfeldt, J. O.; Hook, F. Nano Lett. 2008, 8, 183-188.

(33) Lee, S.; Spencer, N. D. Langmuir 2008, 24, 9479-9488.

(34) Chan, T. F.; Ha, C.; Phong, A.; Cai, D. M.; Wan, E.; Leung, L.; Kwok, P. Y.; Xiao, M. Nucleic Acids Res. 2006, 34, e113.

(35) Paige, M. F.; Bjerneld, E. J.; Moerner, W. E. Single Molecules 2001, 2, 191-201.

(36) Hiller, Y.; Gershoni, J. M.; Bayer, E. A.; Wilchek, M. Biochem. J. 1987, 248, 167-171.

(37) Dukkipati, V. R.; Kim, J. H.; Pang, S. W.; Larson, R. G. Nano Lett. 2006, 6, 2499-2504.

(38) Bensimon, A.; Simon, A.; Chiffaudel, A.; Croquette, V.; Heslot, F.; Bensimon, D. Science 1994, 265, 2096-2098.

(39) Kudo, H.; Suga, K.; Fujihira, M. Colloids Surf., A 2008, 313-314, 651-654.

(40) van Mameren, J.; Peterman, E. J. G.; Wuite, G. J. L. Nucleic Acids Res. 2008, 36, 4381-4389.

(41) Bensimon, D.; Simon, A. J.; Croquette, V.; Bensimon, A. Phys. Rev. Lett. 1995, 74, 4754-4757.

(42) Holmberg, K.; Tiberg, F.; Malmsten, M.; Brink, C. Colloids Surf., A 1997, 123, 297-306.

(43) Petit, C. A. P.; Carbeck, J. D. Nano Lett. 2003, 3, 1141-1146.

(44) Marko, J. F.; Siggia, E. D. Macromolecules 1995, 28, 8759-8770.

(45) Murade, C. U.; Subramaniam, V.; Otto, C.; Bennink, M. L. Biophys. J. 2009, 97, 835-843.

(46) Psaltis, D.; Quake, S. R.; Yang, C. H. Nature 2006, 442, 381-386. BM901453U 